\title{
Theoretical Background and Clinical Aspects of the Use of EMDR in Patients With Bipolar Disorder
}

\author{
Alicia Valiente-Gómez \\ Institute of Neuropsychiatry and Addictions (INAD), Parc de Salut Mar, Centre Forum Research Unit, Barcelona, Spain \\ Hospital del Mar Research Institute (IMIM), Barcelona, Spain \\ Autonomous University of Barcelona, Spain \\ Centro de Investigación Biomédica en Red Salud Mental (CIBERSAM), Madrid, Spain
}

Ana Moreno-Alcázar

Itxaso Gardoki-Souto

Institute of Neuropsychiatry and Addictions (INAD), Parc de Salut Mar, Centre Forum Research Unit, Barcelona, Spain Hospital del Mar Research Institute (IMIM), Barcelona, Spain

\section{Carmen Masferrer}

Sara Porta (10)

Oscar Royuela

Institute of Neuropsychiatry and Addictions (INAD), Parc de Salut Mar, Centre Forum Research Unit, Barcelona, Spain

\section{Bridget Hogg $(1)$}

Institute of Neuropsychiatry and Addictions (INAD), Parc de Salut Mar, Centre Forum Research Unit, Barcelona, Spain Hospital del Mar Research Institute (IMIM), Barcelona, Spain

\section{Walter Lupo}

Institute of Neuropsychiatry and Addictions (INAD), Parc de Salut Mar, Centre Forum Research Unit, Barcelona, Spain

\section{Benedikt L. Amann}

Institute of Neuropsychiatry and Addictions (INAD), Parc de Salut Mar, Centre Forum Research Unit, Barcelona, Spain Hospital del Mar Research Institute (IMIM), Barcelona, Spain

Autonomous University of Barcelona, Spain

Centro de Investigación Biomédica en Red Salud Mental (CIBERSAM), Madrid, Spain

\begin{abstract}
Bipolar disorder (BD) is associated with a lifelong episodic course of severe mood and behavioral disturbance. In last decades treatment improved with numerous pharmacological and psychosocial treatments; however, subsequent mood episode rates are still high and possible risk factors for subsequent mood episodes are not sufficiently addressed. Of note, childhood trauma and stressful life events represent significant, under-recognized, and often neglected environmental risk factors in the etiology and course of BD. Here, we summarize the evidence of eye movement desensitization and reprocessing (EMDR) therapy in BD with posttraumatic stress disorder (PTSD) or life traumatic events. So far, one case report study and one pilot randomized controlled trial (RCT) have been published suggesting positive effect of EMDR therapy in BD. Currently, two larger further RCTs are ongoing to increase scientific evidence of the use of EMDR therapy in this indication, especially with a focus on its effect on relapse prevention. In addition, a functional neuroimaging case report of a bipolar subject versus 30 healthy controls showed first evidence that EMDR might modulate the default mode network. These preliminary results suggest that EMDR could be a promising and safe psychotherapeutic approach for the add-on treatment of bipolar subjects, but confirmative large RCT are needed, with two currently being conducted.
\end{abstract}

Keywords: EMDR; bipolar disorder; psychological trauma; stressful life events; relapse 
B ipolar disorder $(\mathrm{BD})$ is a severe chronic psychiatric disorder where patients suffer from serious mood changes in the form of depressive, hypomanic, manic, or mixed episodes. Furthermore, bipolar subjects also experience behavioral alterations, high-risk behavior, frequent cognitive and functional decline, and increased mortality due to cardiovascular incidents and suicide when compared to the general population (Grande, Berk, Birmaher, \& Vieta, 2015). The etiology of BD is complex and multifactorial, as it involves both genetic and environmental factors. A recent Nature Genetics publication could prove that 30 loci are associated with the etiology of BD (Stahl et al., 2019). Beyond this polygenetic predisposition, childhood adversity has been identified in a recent umbrella meta-analysis as one of the most robust etiological risk factors to develop BD (Bortolato et al., 2017).

Due to the genetic component within BD's etiology, the therapeutic basis of $\mathrm{BD}$ consists of a variety of pharmacological drugs, mainly mood stabilizers such as lithium, anticonvulsants, or atypical antipsychotics, and occasionally of antidepressants, in the case of more severe bipolar depressive episodes. Pharmacological treatment is usually recommended for life to avoid or at least reduce numbers of affective relapses across the lifespan. To reach this therapeutic goal, clinicians and researchers became aware in the early 1990s of the necessity of additional psychosocial interventions such as psychoeducation, family-focused intervention, functional remediation, or mindfulness, among others. They have been increasingly studied and added to the individual treatment plan in order to improve the prognosis of this complex and often refractory disorder (Bonnin et al., 2016; Miklowitz \& Chung, 2016; Oud et al., 2016; Reinares, SánchezMoreno, \& Fountoulakis, 2014; Secades-Álvarez \& Fernández-Rodríguez, 2017).

Despite these advances, many bipolar-I patients (manic alternating with depressive episodes) and bipolar-II patients (depressive alternating with hypomanic episodes) do not have a complete remission (Paykel, Abbott, Morriss, Hayhurst, \& Scott, 2006) and may also suffer from multiple subsequent mood episodes (Radua, Grunze, \& Amann, 2017). Radua et al.'s (2017) meta-analysis of observational international long-term studies included 5,837 bipolar-I and -II patients, and reported that $70 \%$ of bipolar I and $80 \%$ of bipolar-II patients had at least one subsequent mood episode within a 4-year follow-up. They also found that persisting subsyndromal symptoms, which are defined as the presence of minor hypomanic or depressive symptoms without clinical remission, are a dominant risk factor for rapid subsequent mood episodes. Further risk factors include poor adherence to pharmacological treatment, lack of insight, cognitive deficits, or somatic and psychiatric comorbidities. Among psychiatric comorbid disorders, posttraumatic stress disorder (PTSD) has been identified in $16 \%-39 \%$ of bipolar subjects (Otto et al., 2004). The comorbidity between BD and PTSD has been associated with faster cycles, more suicide attempts, more substance abuse, a lower quality of life, and more (hypo)manic and depressive symptoms than bipolar subjects without PTSD (Goodman et al., 2001; Quarantini et al., 2010). Of importance, life events also increase the risk of suffering from more affective episodes in BD (Simhandl, Radua, Konig, \& Amann, 2015).

Therefore, a further psychosocial/psychotherapeutic intervention focused on psychological trauma is needed within the individual treatment plan of bipolar patients. One candidate hereby is eye movement desensitization and reprocessing (EMDR) (Shapiro, 2001), which is an integrative psychotherapy approach using standardized protocols. It includes elements of cognitive-behavioral therapy, interpersonal and bodycentered therapies, as well as dual stimulation in form of mainly horizontal eye movements (Shapiro, 2009; Shapiro, 2001). A recent Nature study, using an animal model, revealed its mechanism of action and neuroanatomic pathway (Baek et al., 2019). EMDR therapy has been recommended by the World Health Organization (2013) for the treatment of PTSD in adults and children and adolescents (World Health Organization, 2013), but its use is currently expanding to the treatment of other conditions with comorbid psychological trauma, such as psychosis, BD, unipolar depression, anxiety disorders, and addiction with promising results (Valiente-Gómez et al., 2017). As a matter of fact, there exists no reasonable argument not to treat a comorbid trauma condition in $\mathrm{BD}$, as it is state of art to treat comorbid anxiety disorders, obsessive-compulsive disorders, or substance use disorders in severe mental disorders. Furthermore, so far randomized controlled trials have shown EMDR therapy to be safe in psychosis or BD with comorbid psychological trauma. In this line, a recent article by experts in the field of $\mathrm{BD}$ suggested that pragmatic clinical treatment options, including those for comorbid conditions, have to be offered for bipolar subjects, even though scientific clinical evidence might still be preliminary (Post, Yatham, Vieta, \& Berk, 2019). 


\section{Clinical Aspects of the Current Status of EMDR in BD}

It must be stated that the current scientific evidence of the use of EMDR therapy in BD is still limited. So far, one case report study (Oh \& Kim, 2014) and one randomized controlled trial (Novo et al., 2014) have been published.

The case report study describes two patients with $\mathrm{BD}$ and comorbid PTSD who were treated with 9 and 10 weekly EMDR sessions, respectively (Oh \& Kim, 2014). Both were assessed with the ClinicianAdministered PTSD Scale (CAPS) before and after EMDR therapy and pharmacotherapy dose was maintained unchanged over EMDR treatment. At the end of the EMDR treatment, both patients achieved full remission of PTSD diagnosis, which was maintained at the 1-year follow-up. EMDR was considered safe in both cases.

In the randomized controlled trial (Novo et al., 2014), 20 bipolar subjects with subsyndromal affective symptoms (see definition above) and a history of traumatic events were enrolled and randomly assigned to EMDR therapy or treatment as usual (TAU). The participants were assessed at baseline, after the treatment, at 12 weeks, and at follow-up visit at 24 weeks. The results of this study showed significant reductions in affective symptom scores, assessed using the Hamilton Depression Rating Scale and the Young Mania Rating Scale, in favor of the EMDR group after treatment. However, changes from baseline to the 24-week follow-up visit did not reach statistical significance. Regarding trauma symptoms, assessed by the CAPS and the Impact Event Scale (IES), results showed a significant improvement in the EMDR group after treatment, in both measures. At the follow-up assessment, only the IES scores remained statistically significant. The EMDR intervention was considered safe, as no adverse events occurred in the EMDR group. The loss of significance in some measures was probably due to the small sample size of this pilot trial. Of note, in this study the Shapiro standard EMDR protocol (2001) was used, with effective results; however, after analyzing in detail the experience of each EMDR therapist, they concluded that a more detailed manual for bipolar subjects would better address the patients' needs due to their often current ongoing stressful events, past complex traumas, traumatic experiences as psychiatric patients, and further comorbidities. Therefore, the same group published a specific EMDR protocol for BD (Amann et al., 2015), which included a summary about the state of art of $\mathrm{BD}$, a detailed inventory to identify traumatic events across clients' lifetime, including also iatrogenic adversities such as mechanical restraint and involuntary admissions or medication, and first affective episodes with corresponding triggers. In addition to the Shapiro standard protocol (Shapiro, 2001), they included the inverted protocol by Hofmann (Hofmann, 2010), and added new five subprotocols to improve via bilateral stimulation mood stabilization, treatment adherence, insight, a faster treatment of prodromal symptoms, and decreasing idealization symptoms of manic episodes. Currently, this protocol is being tested in a single-blind, randomized controlled, multicenter trial (Moreno-Alcázar et al., 2017), in which 82 patients with $\mathrm{BD}$ and a history of traumatic events are randomly allocated to EMDR therapy or supportive therapy (ST). Patients in both groups receive 20 individual EMDR or ST sessions, 60 minutes each during 6 months. The goals of the study are to replicate initial positive preliminary results and to test whether EMDR therapy is effective in the reduction of subsequent mood episodes, affective and trauma-related symptoms, and in the improvement of cognitive performance, social cognition, and functioning after therapy and at 12 and 24 months.

A second randomized controlled trial is currently ongoing in Holland that aims to recruit 36 bipolar subjects with subsyndromal symptoms and a history of traumatic events to test whether or not the EMDR standard protocol (Shapiro, 2001) improves trauma and affective symptoms in this bipolar sample (personal communication Ad de Jongh, 2019).

\section{Can EMDR Modulate Brain Networks?}

One female bipolar patient of our randomized controlled trial (Novo et al., 2014), who received 14 EMDR sessions, underwent a functional neuroimaging ( $\mathrm{fMRI}$ ) session before and after the intervention (Landin-Romero et al., 2013). The subject performed a sequential-letter version of the working memory n-back task, which was compared with 30 healthy subjects. The results showed a normalization of the bipolar patient's default mode network (DMN) after the treatment in terms of activation patterns and especially in the failure of deactivation. The DMN is a neural network that consists of a group of several brain regions that show relative hypoactivity during goaloriented or attention-demanding tasks (WhitfieldGabrieli \& Ford, 2012) and are activated during introspective thinking, remembering personal experiences, making social and emotional judgments, visualizing the future, and performing theoretical tasks 
of the mind. In lay words, if subjects have an abnormal DMN functioning, their attention is too much drawn to the past or the future, while their focus on the present is altered. Interestingly, a failure of deactivation is a typical DMN abnormality in major psychiatric disorders, having been documented in schizophrenia, BD, and unipolar depression (Goikolea et al., 2019; Landin-Romero et al., 2015; RodríguezCano et al., 2017).

Our case fits with structural neuroimaging findings, which have found a normalization of important brain regions such as the medial prefrontal cortex, the amygdala, or the hippocampus after cognitive behavioral therapy, prolonged exposure therapy, or EMDR (Boukezzi et al., 2017; Helpman et al., 2016; Levy-Gigi, Szabó, Kelemen, \& Kéri, 2013). For further details, we recommend a recent review on the interaction of DMN, psychiatric disorders, and childhood adversities (Allen, Sommer, Jardri, Eysenck, \& Hugdahl, 2019).

It is important again to emphasize that evidence of a possible positive modulation of the DMN via EMDR therapy is preliminary with larger studies warranted, but EMDR might have a direct effect on important neurobiological structures and networks implicated in psychiatric disorders and psychological trauma.

\section{Conclusion}

In summary, there is preliminary evidence that EMDR therapy may be useful and safe for the treatment of comorbid PTSD symptoms or psychological trauma in subjects with BD. In addition, it appears to improve not only trauma-related symptoms, but also affective subsyndromal symptoms. Whether or not this intervention also prevents subsequent mood episodes has not yet been determined. The idea of a potential mood stabilizing psychotherapy in $\mathrm{BD}$ is currently being tested in a large randomized controlled trial with a follow-up of 2 years (Moreno-Alcázar et al., 2017). Further trials of other international groups, such as the Dutch randomized controlled trial, are relevant as well, to replicate first positive results. Considering that one of the most robust risk factors in the onset of BD is having suffered from childhood adversities (Bortolato et al., 2017) and that life events worsen the course of BD (Simhandl et al., 2015), it is a clinical necessity to evaluate and attend to adverse events in this disorders. The principal idea is that EMDR therapy is a further additional psychotherapeutic pragmatic tool for traumatized bipolar subjects, which can be added to other interventions such as psychoeducation or cognitive behavior strategies in this complicated to treat population. The implementation of
EMDR therapy in BD clinic units and mental health services may therefore offer a deeper understanding of the relationship between psychological trauma and mental health.

\section{References}

Allen, P., Sommer, I. E., Jardri, R., Eysenck, M. W., \& Hugdahl, K. (2019). Extrinsic and default mode networks in psychiatric conditions: Relationship to excitatory-inhibitory transmitter balance and early trauma. Neuroscience and Biobehavioral Reviews, 99, 90-100. doi:10.1016/j.neubiorev.2019.02.004

Amann, B.L., Batalla, R., Blanch, V., Capellades, D., Carvajal, M.J., Fernández, I., . . . Luber, M. (2015). The EMDR therapy protocol for bipolar disorder. In M. Luber (Ed.), Eye movement desensitization and reprocessing (EMDR) scripted protocols and summary sheets: Treating trauma, anxiety and mood-related conditions (pp. 223-287). New York, NY: Springer Publishing.

Baek, J., Lee, S., Cho, T., Kim, S. W., Kim, M., Yoon, Y., \& . . . Shin, H. S. (2019). Neural circuits underlying a psychotherapeutic regimen for fear disorders. Nature, 566(7744), 339-343. doi:10.1038/s41586019-0931-y

Bortolato, B., Köhler, C. A., Evangelou, E., León-Caballero, J., Solmi, M., Stubbs, B., . . . Carvalho, A. F. (2017). Systematic assessment of environmental risk factors for bipolar disorder: An umbrella review of systematic reviews and meta-analyses. Bipolar Disorders, 19(2), 84-96. doi:10.1111/bdi.12490

Boukezzi, S., El Khoury-Malhame, M., Auzias, G., Reynaud, E., Rousseau, P. F., Richard, E., . . . Khalfa, S. (2017). Grey matter density changes of structures involved in Posttraumatic stress disorder (PTSD) after recovery following eye movement desensitization and reprocessing (EMDR) therapy. Psychiatry Research Neuroimaging, 266, 146-152. doi:10.1016/j.pscychresns.2017.06.009

Garno, J. L., Goldberg, J. F., Ramirez, P. M., \& Ritzler, B. A. (2005). Impact of childhood abuse on the clinical course of bipolar disorder. British Journal of Psychiatry, 186, 121-125. doi:10.1192/bjp.186.2.121

Goikolea, J. M., Dima, D., Landín-Romero, R., Torres, I., Delvecchio, G., Valentí, M., . . . Vieta, E. (2019). Multimodal brain changes in first-episode mania: A voxelbased morphometry, functional magnetic resonance imaging, and connectivity study. Schizophrenia Bulletin, 7;45(2):464-473. doi:10.1093/schbul/sby047

Goodman, L. A., Salyers, M. P., Mueser, K. T., Rosenberg, S. D., Swartz, M., Essock, S. M., . . . Swanson, J. (2001). Recent victimization in women and men with severe mental illness: Prevalence and correlates. Journal of Traumatic Stress, 14(4), 615-632. doi:10.1023/A: 1013026318450 
Grande, I., Berk, M., Birmaher, B., \& Vieta, E. (2016). Seminar bipolar disorder. The Lancet, 387(10027):1561-1572. doi:10.1016/S0140-6736(15)00241-X

Helpman, L., Papini, S., Chhetry, B. T., Shvil, E., Rubin, M., Sullivan, G. M., . . . Neria, Y. (2016). PTSD remission after prolonged exposure treatment is associated with anterior cingulate cortex thinning and volume reduction. Depression and Anxiety, 33(5), 384-391. doi:10.1002/da.22471

Hofmann, A. (2010). The inverted EMDR standard protocol for unstable complex post-traumatic stress disorder. In M. Luber (Ed.), Eye movement desensitization (EMDR) scripted protocols: Special populations (pp. 313-328). New York, NY: Springer Publishing.

Landin-Romero, R., McKenna, P. J., Salgado-Pineda, P., Sarró, S., Aguirre, C., Sarri, C., . . . PomarolClotet, E. (2015). Failure of deactivation in the default mode network: A trait marker for schizophrenia? Psychological Medicine, 45(6), 1315-1325. doi:10.1017/ S0033291714002426

Landin-Romero, R., Novo, P., Vicens, V., McKenna, P. J., Santed, A., Pomarol-Clotet, E., ... Amann, B. L. (2013). EMDR therapy modulates the default mode network in a subsyndromal, traumatized bipolar patient. Neuropsychobiology, 67(3),181-184. doi:10.1159/000346654

Levy-Gigi, E., Szabó, C., Kelemen, O., \& Kéri, S. (2013). Association among clinical response, hippocampal volume, and FKBP5 gene expression in individuals with posttraumatic stress disorder receiving cognitive behavioral therapy. Biological Psychiatry, 74(11), 793-800. doi:10.1016/j.biopsych.2013.05.017

Moreno-Alcázar, A., Radua, J., Landín-Romero, R., Blanco, L., Madre, M., Reinares, M., . . Amann, B. L. (2017). Eye movement desensitization and reprocessing therapy versus supportive therapy in affective relapse prevention in bipolar patients with a history of trauma: Study protocol for a randomized controlled trial. Trials, 18(1), 160. doi:10.1186/s13063-017-1910-y

Miklowitz, D.J., \& Chung, B. (2016). Family-focused therapy for bipolar disorder: Reflections on 30 Years of research. Family Process, 55(3), 483-499. doi:10.1111/ famp.12237

Novo, P., Landin-Romero, R., Radua, J., Vicens, V., Fernandez, I., Garcia, F., . . . Amann, B. L. (2014). Eye movement desensitization and reprocessing therapy in subsyndromal bipolar patients with a history of traumatic events: A randomized, controlled pilot-study. Psychiatry Research, 219(1), 122-128. doi:10.1016/j.psychres.2014.05.012

Oh, D., \& Kim, D. (2014). Eye movement desensitization and reprocessing for posttraumatic stress disorder in bipolar disorder. Psychiatry Investigation, 11(3), 340-341. doi:10.4306/pi.2014.11.3.340

Otto, M. W., Perlman, C. A., Wernicke, R., Reese, H. E., Bauer, M. S., \& Pollack, M. H. (2004). Posttraumatic stress disorder in patients with bipolar disorder: A review of prevalence, correlates, and treatment strategies.
Bipolar Disorders, 6(6):470-9. doi:10.1111/j.13995618.2004.00151.x

Oud, M., Mayo-Wilson, E., Braidwood, R., Schulte, P., Jones, S.H., Morriss, R., . . . Kendall, T. (2016). Psychological interventions for adults with bipolar disorder: Systematic review and meta-analysis. British Journal of Psychiatry, 208(3), 213-222. doi:10.1192/ bjp.bp.114.157123

Paykel, E. S., Abbott, R., Morriss, R., Hayhurst, H., \& Scott, J. (2006). Sub-syndromal and syndromal symptoms in the longitudinal course of bipolar disorder. British Journal of Psychiatry, 189(AUG.), 118-123. doi:10.1192/bjp.bp.105.013870

Post, R. M., Yatham, L. N., Vieta, E., \& Berk, M. N. A. (2019). Beyond evidence-based treatment of bipolar disorder: Rational pragmatic approaches to management. Bipolar Disorders. Advance online publication. doi:10.1111/bdi.12813

Quarantini, L. C., Miranda-Scippa, Â, Nery-Fernandes, F., Andrade-Nascimento, M., Galvão-de-Almeida, A., Guimarães, J. L., . . Koenen, K. C. (2010). The impact of comorbid posttraumatic stress disorder on bipolar disorder patients. Journal of Affective Disorders, 123(1-3), 71-76. doi:10.1016/j.jad.2009.08.005

Radua, J., Grunze, H., \& Amann, B. L. (2017). Meta-analysis of the risk of subsequent mood episodes in bipolar disorder. Psychotherapy and Psychosomatics, 86(2), 90-98. doi:10.1159/000449417

Reinares, M., Sánchez-Moreno, J., Fountoulakis, K.N. (2014). Psychosocial interventions in bipolar disorder: What, for whom, and when. Journal of Affective Disorders, 156, 46-55. doi:10.1016/j.jad.2013.12.017

Rodríguez-Cano, E., Alonso-Lana, S., Sarró, S., FernándezCorcuera, P., Goikolea, J. M., Vieta, E., . . . PomarolClotet, E. (2017). Differential failure to deactivate the default mode network in unipolar and bipolar depression. Bipolar Disorders, 19(5), 386-395. doi:10.1111/bdi.12517

Secades-Álvarez, A., \& Fernández-Rodríguez, C. (2017). Review of the efficacy of treatments for bipolar disorder and substance abuse. Revista de Psiquiatria y Salud Mental (English Edition), 10(2), 113-124. doi:10.1016/j.rpsmen.2017.05.002

Shapiro, F. (2001). Eye movement desensitization and reprocessing: Basic principles, protocols, and procedures (2nd ed.). New York, NY: Guilford Press.

Simhandl, C., Radua, J., Konig, B., \& Amann, B. L. (2015). The prevalence and effect of life events in 222 bipolar I and II patients: A prospective, naturalistic 4 year follow-up study. Journal of Affective Disorders, 170, 166-171. doi:10.1016/j.jad.2014.08.043

Stahl, E. A., Breen, G., Forstner, A. J., McQuillin, A., Ripke, S., Trubetskoy, V., . . . Sklar, P. (2019). Genomewide association study identifies 30 loci associated with bipolar disorder. Nature Genetics, 51(5), 793-803. doi:10.1038/s41588-019-0397-8 
Valiente-Gómez, A., Moreno-Alcázar, A., Treen, D., Cedrón, C., Colom, F., Pérez, V., \& Amann, B. L. (2017). EMDR beyond PTSD: A systematic literature review. Frontiers in Psychology, 6, 1668. doi:10.3389/ fpsyg.2017.01668

Whitfield-Gabrieli, S., \& Ford, J. M. (2012). Default mode network activity and connectivity in psychopathology. Annual Review of Clinical Psychology, 8(1), 49-76. doi:10.1146/annurev-clinpsy-032511-143049

World Health Organization. (2013). Guidelines for the management of conditions specifically related to stress. Geneva: Author.

Disclosure. BLA has been invited to national or international EMDR conferences and has received fees for providing EMDR training. He is also the head of the European EMDR Research Committee. The other authors have no relevant financial interest or affiliations with any commercial interests related to the subjects discussed within this article.
Acknowledgments. This work was supported by a grant from the Plan Nacional de $\mathrm{I}+\mathrm{D}+\mathrm{i}$ and co-funded by the Instituto de Salud Carlos III-Subdirección General de Evaluación y Fomento de la Investigación with a Research Project to BLA (PI/15/02242). BLA received a NARSARD Independent Investigator Award (number 24397) from the Brain and Behavior \& Behavior Research Foundation (24397). We further acknowledge the generous support by the Centro de Investigación Biomédica en Red de Salud Mental (CIBERSAM), Madrid, Spain.

Correspondence regarding this article should be directed to Benedikt L. Amann, Centre Forum Research Unit, Parc de Salut Mar, Hospital del Mar Research Institute (IMIM), Department of Psychiatry, Autonomous University of Barcelona, Calle de Llull 410, 08019 Barcelona, Spain. E-mail: benedikt.amann@gmail.com 